\title{
Prevalence and Side Effects of Energy Drink Consumption among Medical Students at Umm Al-Qura University, Saudi Arabia
}

\author{
Naif A. Bawazeer,' Najmah A. AlSobahi
}

\begin{abstract}
Background: Energy drinks are freely available at markets and shops on the university campus without regulation or proper education regarding its side effects. The caffeine amount within energy drinks is high and could become an addictive substance or cause intoxication. Therefore, this study aims to assess the prevalence of energy drink consumption and its reported side effects among medical students. Methods: A total number of 257 medical students from Umm Al-Qura University completed a questionnaire about energy drinks that was administrated electronically from September through November, 2012. Results: Out of the 257 participants, $27.2 \%$ ( $n=70)$ reported consuming at least one energy drink per month, with $61.5 \%(n=48)$ being males. Males consume significantly more energy drinks than females $(p=0.0001)$. The students consumed energy drinks to get energy in general $(32.8 \%)$ and while studying for exams or finishing a project (31.4\%). Other reasons given include, lack of sleep (12.8\%), just to be like friends (11.4\%), or driving $(8.5 \%)$. Heart palpitations are the most common side effect in our sample (20\%), followed by insomnia (10\%), headache and tremors $(5.7 \%)$, nausea and vomiting $(4.2 \%)$ and nervousness $(2.8 \%)$. Conclusion: Energy drinks consumption is common practice among medical students and the main reason cited for consumption is the need for energy during general activities. Approximately one-third of the consumers manifested some side effect after consumption. We recommend the need to create public awareness about energy drinks. Further studies are recommended to assess the educational level of students consuming energy drinks, about the dangerous side effects.
\end{abstract}

\section{Keywords: Energy Drinks, Caffeine, Adverse effects, Medical Students (Source: MeSH-NLM)}

About the Author: Naif Bawazeer is a recently graduated physician, current ly working as teaching assistant at the Faculty of Medicine, Umm Al-Qura University.

\section{Introduction}

Energy drinks started in 1997 when RedBull ${ }^{\circledR}$ was first released, and followed by many others trade names. With time, energy drinks became more than just drinks, the young population consumed them to indicate they have an athletic and modern lifestyle, while others to increase their cognitive function, especially before exams or an assignment deadlines. Hence, more than 500 new energy drinks had been launched worldwide by 2006 and beverage companies were reaping the financial rewards of the 5.7 billion dollar energy drink industry. ${ }^{2}$

The literature shows that energy drinks give consumers some energizing effects when compared to placebo, as the advertisements claim. ${ }^{3}$ Caffeine is the primary compound responsible for these effects and it is absorbable by all body tissues. Caffeine is structurally similar to adenosine, and its amount in 8 ounces of energy drinks is about 80 to $141 \mathrm{mg} .{ }^{4}$ In addition, previous studies have revealed that high caffeine amounts are able to induce diuresis, insulin resistance and increase mean arterial blood pressure. ${ }^{5}$ Also, high contents of carbohydrates, guarana, taurine, ginseng and creatine play an important role in the energy boosting process. ${ }^{6}$ Furthermore, caffeine can fulfill all the criteria for being an addictive substance, namely dependence, tolerance, and withdrawal.? The symptoms of ca- ffeine withdrawal include nervous irritability, tremors, muscle twitching, sensation disturbances, tachypnea, palpitation, flushing, arrhythmias, diuresis, gastrointestinal disturbances and patients may have generalized anxiety or depression. ${ }^{8}$

Previous studies showed that caffeine has side effects and may reach intoxication levels; studies show that almost every organ system is affected when caffeine is taken in large quantity. ${ }^{9}$ Caffeine intoxication presents with nausea and vomiting, which can be difficult to control, along with agitation, nervousness, headache, tremors, and sleep disturbances. ${ }^{10}$ More serious side effects include tachycardia and arrhythmias, in addition to electrolyte imbalances such as hypokalemia, hypomagnesaemia and hypophosphatemia. Seizures can result from the increased serum lactate, which is preceded by hyperglycemia and metabolic acidosis. ${ }^{12}$ These conditions could result in a medical emergency. The clinical presentation typically resolves between 4 to 6 hours after intoxication. " On the other hand, the severity of symptoms has been reported to be less than that resulting from intoxication induced by caffeine directly from medications. ${ }^{9}$

Energy drinks are usually stored next to sport drinks in supermarkets and people may confuse them. In fact, sports drinks 
are beverages that aim to replace water and electrolytes lost through sweating during exercise, while energy drinks aim to induce performance-enhancing effects with nonnutritive stimulants that actually result in more dehydration. ${ }^{15}$

Energy drinks are freely available at markets and shops in university campus without regulation or proper education for its side effects for the general public, and more studies are needed to investigate the safety profile among consumers. ${ }^{13,14}$ Therefore there is a great concern about energy drink consumption, especially among the young adult population. Previous studies conducted worldwide among college students and medical school students demonstrated that energy drink consumption is a common practice. However, all of them were observational studies that reflect the pattern and frequencies of consumption only. Moreover, in Saudi Arabia there are no previous studies exploring this uprising problem in the general public or among medical students.

The primary aim of this study was to estimate prevalence of energy drink consumption among Umm Al-qura University medical students and report the side effects present in this population. In addition, the study investigated reasons promoting the use of energy drinks and the beneficial effects after consumption.

\section{Methods}

In this study, 257 Umm Al-Qura University (Makkah, Saudi Arabia) medical students from different stages of the clinical years (4th, 5th and 6th years) participated in this cross-sectional survey, from September to November, 2012. The medical school at Umm Al-Qura University is from the public sector and students are enrolled from all over the country. A self-administered questionnaire was used to collect the data. We distributed the questionnaire electronically as a Google form $\odot$ through social media website groups which covered all students from the clinical years. Invitation requests reached all 750 clinical years students, but there is no guarantee that all reviewed the invitation within the study period. Only 257 out of 750 clinical year students in Umm Al-Qura University completed the survey, for a response rate of $34.3 \%$. The questionnaire was designed after reviewing selected related papers. ${ }^{13-15}$

The questionnaire can be found in the Appendix. Firstly, students were asked their gender, study year, and if they are consuming energy drinks. If they answered affirmatively, they would be prompted with the rest of the questionnaire. The questionnaire evaluated when they started consuming energy drinks, consumption pattern (frequency, quantity and type of energy drinks), reasons for consuming such drinks, if they feel more alert when having these beverages, if they experienced side effects and of which type: heart palpitations, headaches, crash episodes, insomnia, tremor, nervousness, nausea and vomiting and other (specify). Data was entered into SPSS software ${ }^{\circ}$ (version16). The chi-square test was used to assess associations between variables and a value of $\mathrm{P}<0.05$ was taken as statistically significant with a confidence level of $95 \%$.

Permission from the Ethics committee at the University was granted prior to the initiation of this study. This study follows the STROBE guidelines for cross-sectional studies.
Table 1. Characteristics of Study Participants $(\mathrm{N}=257)$

\begin{tabular}{|lr}
\hline Characteristic & n (\%) \\
\hline Age & \\
\hline 21 & $34(13.2)$ \\
\hline 22 & $92(35.8)$ \\
\hline 23 & $85(33.1)$ \\
\hline 24 & $40(15.6)$ \\
\hline 25 & $6(2.3)$ \\
\hline Cender & $78(30.4)$ \\
\hline Male & $179(69.6)$ \\
\hline Female & $107(41.6)$ \\
\hline Current year of education (clinical years)* & $72(28.0)$ \\
\hline 4 th year students & $78(30.4)$ \\
\hline 5th year students & \\
\hline 6 th year students &
\end{tabular}

Legend: Current year of education in a 6-year program.

Figure 1. Prevalence of Energy Drink Consumption among Medical Students in Clinical Years.

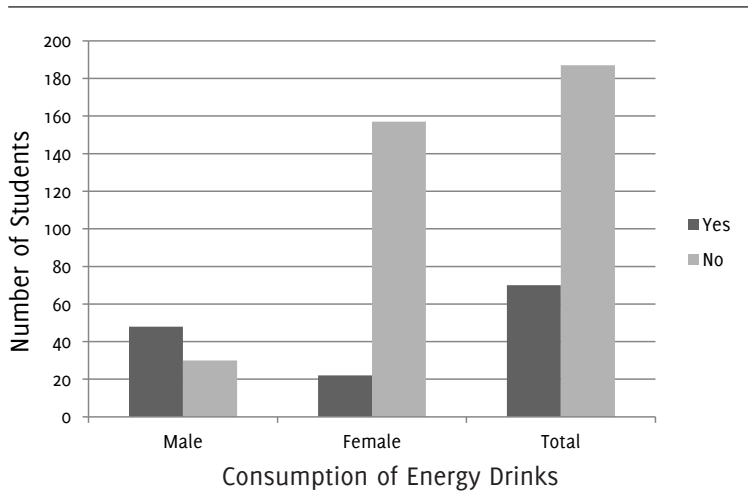

Table 2. Pattern of Consumption among Medical Students $(\mathrm{N}=70)$.

\begin{tabular}{lr}
\hline Pattern of Consumption & $\mathbf{n}(\%)$ \\
Start of Energy Drink Consumption & $14(20.0)$ \\
\hline Since joining med school & $5(7.1)$ \\
\hline 1 year ago & $10(14.3)$ \\
\hline 2 years ago & $41(58.6)$ \\
\hline I don't remember & \\
\hline Frequency of Consumption & $8(11.4)$ \\
\hline Daily & $13(18.6)$ \\
\hline Weekly & $5(7.1)$ \\
\hline Monthly & $44(62.9)$ \\
\hline Occasionally & \\
\hline Type of Energy Drink & $35(50.0)$ \\
\hline Regular & $50(42.9)$ \\
\hline Sugar free &
\end{tabular}


Figure 2. Reasons for Energy Drink Consumption among Medical Students in Clinical Years.

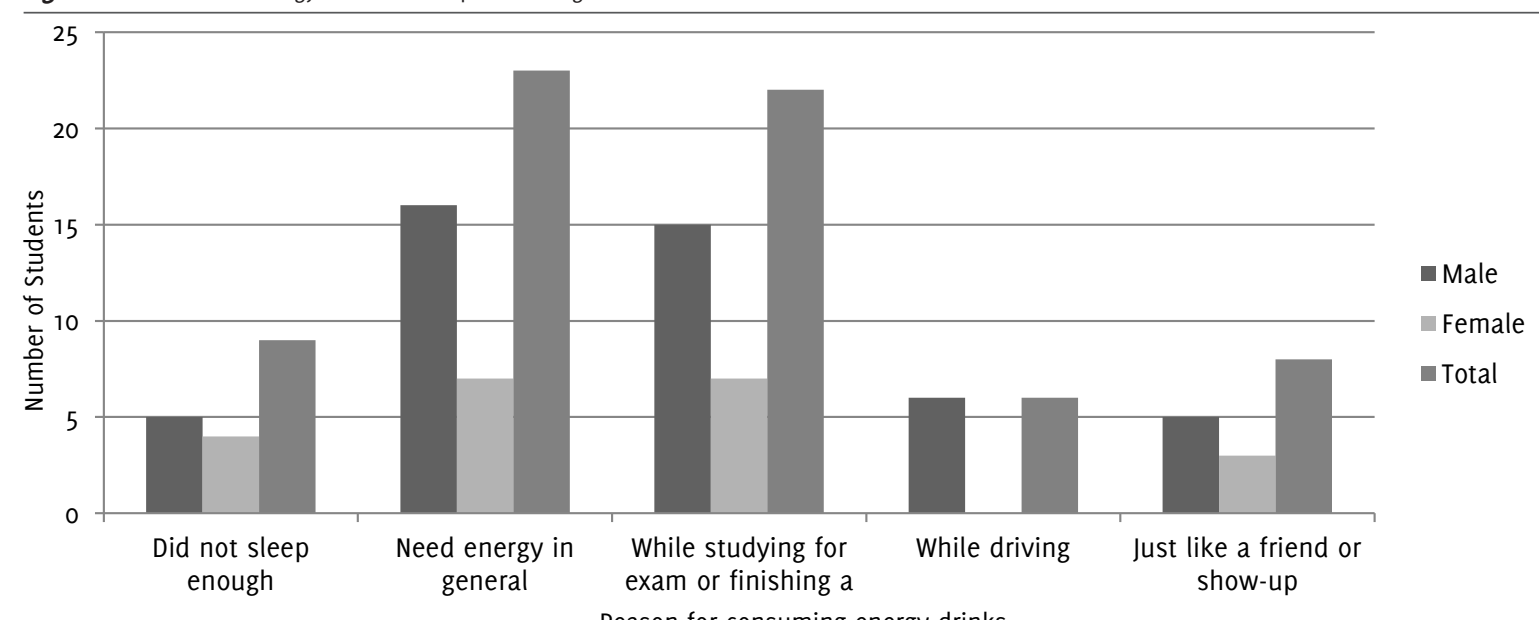

\section{Results}

A total of 257 participants (78 male, 179 female), aged $22.58 \pm$ 0.98 years (ranging fromf 21 to 25 years) answered the questionnaire (Table 1). The total percentage of participants who consumed energy drinks was $27.2 \%(n=70)$ of all students (Figure 1). Regarding the gender of consumers, a significantly higher proportion of male students $(61.5 \%$ of all male students, $\mathrm{n}=48$ ) consumed energy drinks when compared to females (12.3\% of all female students, $n=22, p=0.0001$ ). The pattern of consumption among medical students is presented in Table 2. In the clinical years, the percentage of students that consumed energy drinks was $50.0 \%$ among 4 th year students $(n=35)$, $27.1 \%$ among 5 th year students $(n=19)$ and $22.9 \%$ among 6th year students $(n=16)$. Among consumers of energy drinks, $71.4 \%$ used them once daily, $17.1 \%$ used them twice daily, and $1.4 \%$ drank more than 3 bottles daily.

The reasons for consuming energy drinks (Figure 2) were because they didn't get enough sleep ( $12.8 \%$ of consumers, $n=9$ ), because they needed energy in general $(32.8 \%$ of consumers, $\mathrm{n}=23$ ), to study for an exam or finishing a project $(31.4 \%$ of consumers, $n=22)$, to drive a car for a long time ( $8.5 \%$ of consumers, $n=6$ ) or to be like friends and show-off ( $11.4 \%$ of consu- mers, $n=8)$. This study shows that $44.2 \%$ of consumers $(n=31)$ feel more alert when they drink, $21.4 \%$ of students $(n=15)$ don't feel more alert when they drink, and $34.2 \%$ of consumers $(n=24)$ were not sure.

The questionnaire also showed that $27.1 \%$ of male $(n=13)$ and $50.0 \%$ of female $(n=11)$ consumers of energy drinks manifested some side effect after consumption. Regarding the symptoms experienced (Figure 3 ), $20 \%$ of consumers ( $n=14$ ) experienced heart palpitations, $5.7 \%$ of consumers $(n=4)$ had headaches, $10 \%$ of consumers $(n=7)$ reported having insomnia, $5.7 \%(n=4)$ had tremor, $2.8 \%(n=2)$ reported nervousness and $4.2 \%$ of consumers $(n=4)$ reported nausea and vomiting. Finally, no consumer reported crash episodes as a side effect of energy drink consumption.

\section{Discussion}

Over the past ten years expansion of energy drink marketing has been noted over the world in the absence of clear regulations. ${ }^{13}$ The present study was aimed at estimating the percentage of students consuming energy drinks among Umm Al-Qura university medical students in clinical years and the difference between male and female consumption patterns. We

Figure 3. Reported Symptoms of Caffeine Intoxication after Energy Drink Consumption among Medical Students in Clinical Years.

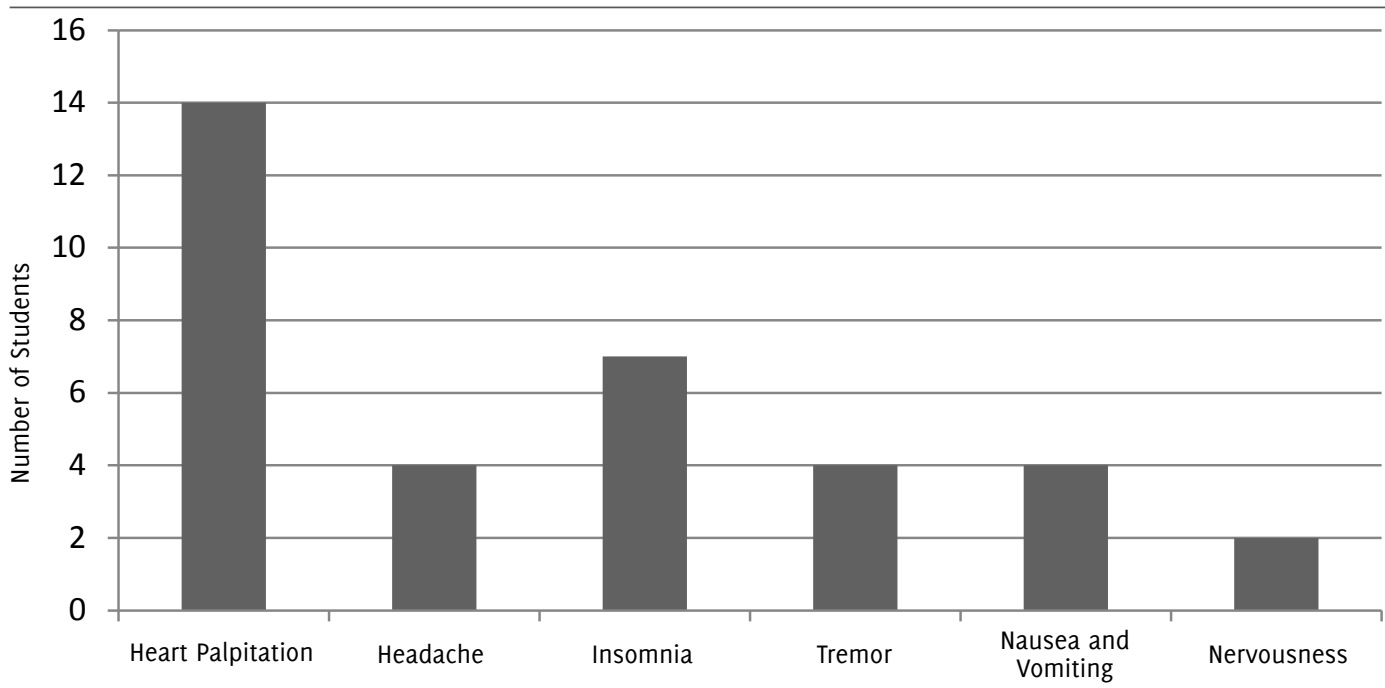

Reported Side Effects 
found that approximately one-third of this sample consumes energy drinks. In an earlier similar study in another medical school in Turkey, the prevalence reported was $32.6 \% .^{13}$ On the other hand, similar studies conducted in non-medical college students shows that around $51 \%$ and $48.3 \%$ of students consume energy drinks. ${ }^{15,16}$ Moreover, a study among university student-athletes showed a prevalence of $62.2 \%$.' This difference in consumption among college versus medical students may be explained by a more extensive understanding of human physiology and toxicology, or cultural differences among different regions of the world. On the other hand, athletes have the desire to improve performance and experience the energetic effect of these beverages, and consequently student-athletes are more prone to consume energy drinks compared to other students, as suggested by Paddock. ${ }^{17}$

Regarding the gender of consumers, we observed that males consume much more frequently than females. In the Middle Eastern culture, females generally are not interested in products that represent an athletic, modern lifestyle according to the general prevailing opinion, which could explain the higher proportion of male consumers in this particular study. In addition, differences in total sample numbers of males and females in this study ( 179 females vs 78 males) could have introduced some bias. These findings are similar to those reported for college students in a southern plains university in 2011, where there was a higher mean consumption in males compared to females. ${ }^{16}$ However, another study performed on students from an university in the central atlantic region of the United States reported a significantly higher proportion of female consuming energy drinks compared to males (53\% vs. $42 \%$ ). ${ }^{15}$ These apparently contradictory results may reflect cultural differences or be the product of other confounders that need to be evaluated in further studies. This will allow further insight into the interesting relationship of gender and energy drink consumption.

The young usually consume energy drinks to benefit from their energetic effects. Nutritionists and health educators should provide education to this population on gaining these effects through healthy diet and exercise. In our study group the majority of students consumed energy drinks for general energy and while studying for an exam or finishing a project. In another context, another study showed similar reasons why students consumed energy drinks: to increase their energy (65\%), to study or complete a major course project $(50 \%)$, and while driving a car for a long period of time $(45 \%) .{ }^{15}$ In Saudi Arabia, females are not allowed to drive a car, which explains why none of the female consumed energy drinks for this particular reason. Other major motivations mentioned in the literature are consuming these beverages mixed with alcohol to become inebriated, to reduce the depressive and sedating effects of alcohol compared to alcohol alone, or to add a new flavor to the mixture..$^{18} \mathrm{~A}$ web-based survey in 2006 reported that $24 \%$ of the participants mixed alcohol with energy drinks within the last month in the United States. ${ }^{19}$ Mixing alcohol with energy drinks may leads to serious events as the mixture is very dehydrating and can reduce the excretion rate for alcohol from the body leading to alcohol in toxication and respiratory depression. ${ }^{8}$ Moreover, it will make the consumer drink more alcohol as he cannot recognize if he had enough alcohol or not. ${ }^{20}$ In the Saudi culture, alcohol consumption is prohibited, and therefore our study did not investi- gate the mixing of alcohol with energy drinks.

Caffeine is the main component in energy drinks that gives a stimulating effect for cognitive function. Its amount in energy drinks exceeds the necessary amount to enhance performance, and could reach intoxication levels if consumers drank more than one bottle per serving. ${ }^{3}$ We report that the most frequent side effect experienced in our sample was heart palpitations, and to a lesser degree headaches, insomnia, tremor, nervousness, nausea and vomiting, and these side effects were more frequent in females than males. Another side effect of consumption are crush episodes. There were no crush episodes reported in this study. Crush episodes are usually reported in athletes during exercise or a match. Investigators have suggested that the large amount of caffeine and taurine consumption together with strenuous exercise can cause coronary vasospasm with an increase in heart rate and blood pressure. In general, side effects do not prevent students from consuming, and percentages of these side effects in our study are almost similar to those reported in a study among college students published in 2007..$^{15}$

We recommend the promotion of public awareness about the active ingredients of energy drinks and the potential side effects and adverse events from reaching intoxication levels. Further studies are needed to evaluate factors involved in consumption of energy drinks among medical students and to explore the relation of gender and consumption, their understanding of risks involved and possible interventions to promote safe consumption.

There are several limitations in this study. First, response rate was low (34.3\%) and this may have introduced some nonresponse or voluntary response bias. This could be reflected in the differing number of female and male participants. The response rate could have been improved by alternate scheduling to conduct the survey with suggestions from direct communication with the participants, allowing more time to respond to the invitation, or offering benefits from participating, among others. Authors limited the number of questions to facilitate that survey would be completed. This study offers important exploratory findings for medical student consumption of energy drinks in Saudi Arabia. Future studies could attempt a different study design to gain more insight into factors contributing to energy drink consumption.

\section{Conclusion}

Energy drinks consumption is common practice among medical students. Out of the 257 medical students who participated in this research, 70 students were consuming energy drinks $(27.2 \%), 61.5 \%(n=48)$ were males and $12.3 \%(n=22)$ were female. Students consumed energy drinks for different reasons, mainly the need for energy in general activities. About one-fifth of the participants manifested some side effect after consumption; heart palpitations were the most frequent side effect reported. We recommend the need to create public awareness about the active ingredients in energy drinks and the potential side effects and adverse events. Further studies are recommended to evaluate factors involved in consumption of energy drinks among medical students, and their understanding of the risks involved as well as possible interventions to promote safe consumption. 


\section{References}

1. Buxton C, Hagan JE. A survey of energy drinks consumption practices among student -athletes in Chana: lessons for developing health education intervention programmes. J Int Soc Sports Nutr. 2012;9(1):9.

2. Boyle M, Castillo VD. Monster on the loose. Fortune 2006;154:116-22.

3. Smit HJ, Cotton JR, Hughes SC, Rogers PJ. Mood and cognitive performance effects of "energy" drink constituents: caffeine, glucose and carbonation. Nutr Neurosci. 2004;7(3):127-39.

4. Smit HJ, Rogers PJ. Effects of low doses of caffeine on cognitive performance, mood and thirst in low and higher caffeine consumers. Psychopharmacology. 2000;152(2):167-73.

5. Lee S, Hudson R, Kilpatrick K, Graham TE, Ross R. Caffeine ingestion is associated with reductions in glucose uptake independent of obesity and type 2 diabetes before and after exercise training. Diabetes Care. 2005;28(3):566-72. 6. Committee on Nutrition and the Council on Sports Medicine and Fitness.. Sports drinks and energy drinks for children and adolescents: are they appropriate? Pediatrics. 2011;127(6):1182-9.

7. Diagnostic and statistical manual of mental disorders. 4th edition text revision. Washington, DC: American Psychiatric Association. 2000;22:150-79. 8. The Tenth Revision of the International Statistical Classification of Diseases and Related Health Problems - 10 classification of mental and behavioral disorders. World Health Organization. 2006;1:3-9.

9. Seifert SM, Seifert SA, Schaechter JL, Bronstein AC, Benson BE, Hershorin $E R$, et al. An analysis of energy-drink toxicity in the National Poison Data System. Clinical Toxicol. 2013;51(7):566-74.

10. Bonnet MH, Balkin TJ, Dinges DF, Roehrs T, Rogers NL, Wesensten N]; Sleep Deprivation and Stimulant Task Force of the American Academy of Sleep Medicine. The use of stimulants to modify performance during sleep loss: a review by the Sleep Deprivation and Stimulant Task Force of the American Academy of Sleep Medicine. Sleep. 2005;28(9):1163-87.

11. Nawrot P, Jordan S, Eastwood J, Rotstein J, Hugenholtz A, Feeley M. Effects of caffeine on human health. Food Addit Contam. 2003;20(1):1-30.

12. Heckman MA, Weil J, Conzalez de Mejia E. Caffeine (1, 3, 7-trimethylxanthine) in foods: a comprehensive review on consumption, functionality, safety, and regulatory matters. J Food Sci. 2010;75(3):R77-87.

13. Hidiroglu S, Tanriover 0 , Unaldi $S$, Sulun S, Karavus M. A survey of energy-drink consumption among medical students. J Pak Med Assoc. 2013;63(7): 842-5.

14. Miller KE. Wired: energy drinks, jock identity, masculine norms, and risk taking. J Am Coll Health. 2008;56(5):481-9.

15. Malinauskas BM, Aeby VG, Overton RF, Carpenter-Aeby T, Barber-Heidal K. A survey of energy drink consumption patterns among college students. Nutr J. 2007;6:35.

16. Pettit ML, DeBarr KA. Perceived stress, energy drink consumption, and academic performance among college students. I Am Coll Health. 2011;59(5):335-41.

17. Paddock R. Energy drinks' effects on student-athletes and implications for athletic departments. The Sport Journal. 2008;11(4).

18. O'Brien MC, McCoy TP, Rhodes SD, Wagoner A, Wolfson M. Caffeinated cocktails: energy drink consumption, high-risk drinking, and alcohol-related consequences among college students. Acad Emerg Med. 2008;15(5):453-60. 19. Marczinski CA. Alcohol mixed with energy drinks: consumption patterns and motivations for use in U.S. college students. Int J Environ Res Public Health. 2011;8(8):3232-45.

20. Attila S, Çakir B. Energy-drink consumption in college students and associated factors. Nutrition. 2011;27(3):316-22.

\section{Acknowledgments \\ The authors are grateful to participants from Umm Al-Qura University for their assistance.}

Conflict of Interest Statement $\mathrm{ct}$ Funding

The Authors have no funding, financial relationships or conflicts of interest to disclose.

Cite as:

Bawazeer NA, Alsobahi N. Prevalence and Side Effects of Energy Drink Consumption among Medical Students at Umm Al-Qura University, Saudi Arabia. Int J Med Students. 2013;1(3):104-8. 